\title{
ALGUNAS ESPECIES DE Bipolaris Y Curvularia protuberata EN GRAMINEAS SILVESTRES DEL NORTE DE ITALIA (LOMBARDIA)
}

\section{Some species of Bipolaris and Curvularia protuberata in wild gramineae from northern Italy (Lombardia)}

\author{
Anna M. Picco*, Edoardo Piontelli**, Marinella Rodolfi*, Valia Vivar** \\ * Universitá degli Studi di Pavia, Facoltá di Scenze Mat. Fis. Nat,Dipartimento di Ecologia del \\ Territorio e degli Ambienti Terresiri, Sezione di Micologia, Via S. Epifanio 14, 27100 PAVIA (Italia). \\ * Universidad de Valparaíso, Escuela de Medicina, Cátedra de Micología, Casilla 92 V, Valparaíso (Chile).
}

Palabras clave: Gramineas silvestres, Bipolaris spp., (Coch/obolus spp.), Curvuhura protuberutu, biogeografia. Key worls: Wild gramineae, Bipolaris spp.,(Cochiobolus spp.), Curvalaria protuberata, biogeography.

\section{RESUMEN}

Entre mayo y noviembre de 1996, se determinó la presencia y distribución geográfica de especies fingicas integrantes del género Curvularia y el rcomplex Helminhosporium!, en las principales gramineas silvestres presemes en t zonas agricolas de la Lombardia (Italia), adyacentes a las ciudades de Milano, Bergano. Crenona y Voghera.

En 18 de las 21 especies de gramineas colectadas, se obturieron 79 aistanientos fungicos. Más del 28\% de éstos, se presentaron como anamorfos de Cochlioholus y los más representatios fincron: Bipolaris anstralicusis $(30,4 \%)$, C.sativus $(27,8)$ y C.cynodontis $(22,8)$. En loghera y Cremona se obmieron los más altos porcentajes de aislamientos fingicos(33-29\%) y en Milamo los menores (13\%). Las espigas y las liojas fneron las más afectadas por la colonizacion fingica, especialmente en verono y el chlmo on oroño. Consideranclo la colonización de las grámineas en el hiempoespacio y el nimero de aislamientos fingicos, se determinoron pumtos iemporo-espociales, créandose 3 grupos de grados de colonización fingica $(A, B, C)$. El grupo A soportó la menor colonización y el C la mayor: En esie illimo, Cynodon doctyton, Hordemm mumimm y Pon pratensis, fueron las gramineas más represemalivas. Se comentan shs potencialidades infestantes y como resemorio de patógenos fungicos de interis agricola.

\section{INTRODUCCION}

Las gramineas cullivadas o silvesires (Poaceac), son una gran familia de Monocotiledoneas que incluye más cle

\section{SUMMARY}

Within may and november of 1996 , the presence and biogeographical distributions of fungal species belonging to the gemus Curvularia and the ucomplex Helminthosporium? were established in the main wild graminea present in 4 agricultural zones of Lombardia (Ilaly) next to the cities of Milano, Bergamo, Cremona and loghera

Trom the 21 gramineae collected, 79 fingal isolafion were obtained in 18 of them. More than $98 \%$ of the isolated species presented as anamorphs of Cochliobolus, being Bipolaris australiensis $(30,4 \%), C$. sativas $(27,8)$ and C.cynodontis $(22,8)$ the most representative. The lighest porcentages of fingal isolates were found in loghera and Cremona(33-20\%) whereas the lowest in Milano(13\%). Ears and leanes were the most afected by the fungal colonization, mainly in summer whereas the culm became affected in attmm. Considering the colonies of gramineae in time-space and in the number of fungal isolates, 3 group $(A, B, C)$ were created according to their degree of fingal colonization together will the establishment of sometime space points. Gronp A shoved the least colonization, B the inteinadiate and C the highest. th the latter; Cynodon dactylon, Hortemm muminum and Poa pratensis, were the most representative gramineae. The potential copabihy of these infecting gromineae as a reservoir of fungal pahogens having an agrarian interest is discussed.

5000 especies vegetales, mayoritariamente herbáceas, anuales o peremes, que adquieren gran importancia al conferir las lípicas características de los paisajes de praderas, pastizales, pampas y savanas. 
Entre los hongos que colonizan gramíneas silvesw ires o cultivadas en sus etapas de desarrollo o senescencia, los dematiáceos representan uno de los mayores grupos de saprotrofos o parásitos. Entre ellos, los representantes de los géneros Cochliobohus, Setosphineriay Pyrenophora y sus anamorfos Bipolaris, Curvularia, Excrohihm y Drechstert, mayoritariamente integrantes del género Irehminthosporium sensu lato, wn nombre gencrico muy conservativo y empleado aún en la lieralura por los firopalólogos (Alcorn, 1983; Korf, 1991). Estos taxa. son capaces de colonizar las raíces, tallos, hojas e innorescencias de estos regchales que crecen desde las zonas tropicales y templadas a las polares, a nivel de mar y lasta en las altas montunas (Alcom, 1982; Lam, 1982; Thomas \& Shatiock. 1986; Sivancsm, 1987: Sisiema, 1987; Liljeroth ei al, 1996).

En cspccial, las gramineas infestantes, favorecidas por una celtaordinaria capacilad adaptativa frente a las varialas condiciones cológicas y agronómicas, pueden cjercer múlliples efecios negalivos sobre has plantas cullivadas. considerando: la competencia frenc a los recursos limitados del ambicntc ta producción de substancias toxicas y hasibilidad de comentirse on potenciales reserrorios de agentes füngicos fitopatogenos (Sholly et al. 1982; Nuller 1982; Lom. 1983; Sonoda. 1991; Carson, 1998). Eslos cfecios pucelen representir una amenaza real o polencial para las áreas de cullivos circundanics, no sólo por su capacidad invasiva y compctitiva, sino por ser agenics naturales de transmision y dispersion de hongos fitopatógenos capaces de infectar un alto mimero de otras gramineas silvesircs o cullivadas. cuando las condiciones climáticas y colificas son fironbles (Widdommih. 1986; Salvinder el al., 1990).

En Intia, la crencision y rotución de cicrios cullivos sobre nucyas superficies en ambientes con diferenlos caracteristicas agrodimáticas, ha inducidon la sclocción de culivares menos susceptibles frente a cienos fitopalógenos talcs como: Pyrenophora teres cn la ceba-

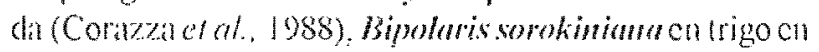
varias regiones de la penimsula (Montorsi a al, 1088: Rossi a al, 1991: Coppolli ar al. 1993; Capcli. 1993) y Bipolaris aryate on arro\% (Molati, 1989, Moldit of at. $1988-92,1096)$

La presencia del "complex Helminthosporinn?" se ha reportado on gramincas formicns o cn sus scmillas. porvarios antores en diversis regiones de Italia (Goviet at. 1974; Cappelli. 1991), incluso se ha descrilo mat nucyal especie (Drechstera pallida) palógena para Sorrehmm halepense (Poria-Puglia \& Del Serronc, 1991). Sincmbirgo, no existe información detallada de su presenciay estacionalidad en las principales gramincas silsesires cr cl norte de la península.

Nuestro objetivo principal. fue elcemimar la presencia y distribución gengrifica le especies lüngicas integrantes de los tava amamórficos Bipolaris, Cursultrita.

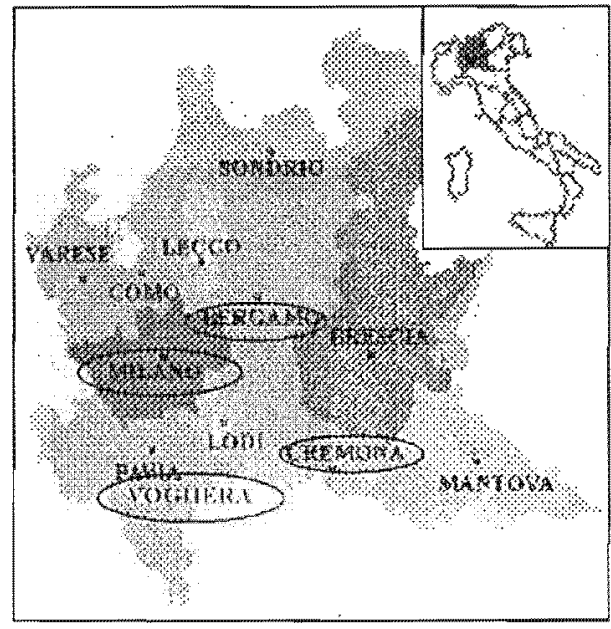

Figura 1.- Región de la Lombardia y zonas de muestrco

Drechstera $y$ Exerohilum $\mathrm{cn}$ gramineas silvestres en 4 zonas de la Lombardia (ltalia del Norte). Como mestros objetivos tuvicron la fimalidad de detectar sólo la presencia o colonización de este gnupo especifico de hongos. no se consideró ha existencia o ausencia de manchas foliares u otras lesiones en tallos, hojas e inforescencins de csias grimineas.

\section{MATERIALES Y METODOS}

a) Recolección de las gramíncas. Entre mayo y noviembre del año 1996 , en + zonas de la Lombardia: Voghera (Pavia), Milano. Bergamoy Cremona (Fig. 1). se efecharon 6 muesircos cstacionales ( 2 en primanera, $2 \mathrm{cn}$ verano y 2 ch olono) de has principales gramincas csponlancas presentes on las zonas agricolas adyacones a las cindades mencionadas (conre 5 a $8 \mathrm{~km}$ de distancia). Se cxcluyeron del discrio muestral, los meses imcmales, por lit auscheia parcial o tolat de las fructificaciones en estos regetales, sinnción que dificulla su determinación.

Paral a recoleceión de las mucsiras, se consideraron ireas de aproximadimonc un $\mathrm{km}^{2}$. seloccionándose principalmone senderos y yonas verdes no cullivadas; descintundose lodos los Ierrenos adyacentes il caminos pavimentados. En un recorriclo de aproximadamente un kilómetro lincal, se recolcctaron a cada lado de esin linca imaginaria las diversas gramincas más commones que se presentaban y' se dispusicron cn bolsas de polictileno eslériles $(30 \times 50 \mathrm{~cm})$. Lis laxade mor fologia semejnme so jumtaron en ma mismi bolsa. previo al corte basal de sus uallos con un cuchillo que se limpinba con solución de yodo elespues de cadia mucsira. Al rimal del recorrido. cada bolsa conchia aproximadianchte chire 30 a 50 cicmplares de curdal especie de gramineir.

Los 2 muesircos en cala cslación se realizaron on las mismas abers, seleccionndose las mismas graimmeas. 
Tabla 1.- Datos metereológicos de los lugares de muestreo considerando (los meses por estación (1996)

\begin{tabular}{|c|c|c|c|c|c|}
\hline & & BBERGAMO & $\mathbb{C R E M O N A}$ & MILANO & VOGHERA \\
\hline \multirow{3}{*}{ PRIMAVERA } & $T^{\circ}$ media $\mathbb{C}^{\circ}$ & $16.0-18.7$ & $19.9-20.5$ & $19.9-22.3$ & $18.1-25.5$ \\
\hline & $H R$ media $\%$ & $64.0-72.0$ & $67.5-69.5$ & $43.5-41.0$ & $66.5-70.0$ \\
\hline & Pliviosilluil non & 13.0 .87 .6$. & $63.9-51.2$ & $65.4-44.8$ & $57.6-71.6$ \\
\hline \multirow{3}{*}{ VERANO } & $T^{\circ}$ media $\mathbb{C}^{\circ}$ & $16.4-23.6$ & $21.5-13.3$ & $26.8-27.4$ & $21.1-27.1$ \\
\hline & $H R$ medice \% & $58.0-65.5$ & $65.0-80.0$ & $46.5-71.0$ & $70.0-76.0$ \\
\hline & Plurosivirl nun & $162.0 .990 \%$ & $29.0-13.0$ & $3.2-11.0$ & $14.3-10.1$ \\
\hline \multirow{3}{*}{ OTOÑO } & $\mathrm{T}^{\circ}$ media $\mathrm{C}^{\circ}$ & $12.6-7.25$ & $13.3-6.1$ & $16.2-9.25$ & $13.2-7.8$ \\
\hline & $H R$ mediu \% & $49.0-60.5$ & $65.0-67.0$ & $70.0-62.5$ & $8 \overline{7.2-76.5}$ \\
\hline & Pluv Viosillad m m & $123.1,11$ & $26,00-5010$ & $86.6-88.0$ & $45.8-125.0$ \\
\hline
\end{tabular}

Las muestras fueron coletadas en los meses de: Mayo y Junio (primavera); Julio y Agosto (verano); Octubre y Noviembre (otoño).

Las gramíneas se conservaron refrigeradas $\left(4-6^{\circ} \mathrm{C}\right)$ en el laboratorio, durante 1 o 2 dias, liempo suficiente para su identificación (2l especies) y posterior preparación para la búsqueda de su micola.

Se colectaron las siguientes gramíneas:

1.- Agropyron repens Beauv.

2.- Alopecurus pratensis L.

3.- Arryenatherum elatium Presl.

4.- Avena fatua L.

5.- Brachypodium pinnatum Beauv.

6.- Bromils arvensis L.

7.- Bromus gussonei Parl.

8.- Bromus slerilis L.

9.- Cynodon dactylon Pers.

10.-Dactylis glomerata L.

11.-Digitaria sanguinalis Scop.

12.-Echinochloa crus-galli Beauv.

13.-Holcus lanatus L

14.-Hordermin murinum L.

15.- Lolium multiflortum Lam.

16.-Lolinm perenne $\mathrm{L}$.

17.-Poa annua $\mathrm{L}$.

18.-Poa pratensis L.

19.-Setaria viridis Beauv.

20.-Sorghum halepense Pers.

21.-Tragus racemosus All.

b) Medios de cultivos. Del material vegetal de cadal bolsa (21), se seleccionaron al azar 4 especímenes, los que se lavaron en agua estéril durante 1 minuto y' luego se seccionaron en trozos de $3-5 \mathrm{~cm}$ para cliferenciar clara- mente culmos, lojas e inflorescencias, las que se depositaron, para el efecto de lograr un cultivo en cámara luúmeda, aséplicamente en duplicado sobre la superficie de placas de Petri de $15 \mathrm{~cm}$ con agar agua (A.A).

Las placas se incubaron a la temperatura ambiente del laboratorio ( 19 a $27^{\circ} \mathrm{C}$ según la época del año) durante un período de 30 días. Cada 10 días y bajo la lupa estereoscópica, se revisaron las placas de Petri en búsqueda de la micota específica planteada en los objetivos. Se efectúaron subcultivos en agar papa dextrosa (PDA), para la conservación de las cepas.

Para evitar las variaciones morfológicas que se presentan en este grupo en cultivo, las determinaciones taxonómicas se efectúaron analizando las estrucluras presentes en el sustrato (culmo, hoja c inflorescencia) en cámara húmeda (AA), los que se clasificaron mediante la literatıra especializada, principalmente: Ellis, 1971, 1976; Alcorn, 1983,1990,1991; Sivanesan 1987.

La presencia de una determinada especie, en cada estructura vegetal considerada se contabilizó una sola vez, aunque sus integrantes se presentaran repetidamente en la misma muestra o en el duplicado. Los resultados de los 2 muestreos estacionales, se sumaron en un solo total por estación.

c) Agrupaciones. Considerando la presencia de grámineas colonizadas en el liempo y el espacio, se determinaron los puntos temporo-espaciales (PTE) combinándolos con los números de aislamientos fúngicos. Se crearon 3 grupos de grados de colonización fúngica de las gramíneas ( $\mathbf{A}, \mathbf{B}, \mathbf{C}$ ) (Fig .2), considerando al grupo $\mathbf{A}$, el que soporta la menor colonización, el B de colonización intermedia y el $\mathbb{C}$ de mayor colonuzación. 
Cuadro 1.- Asociación fúngica: Número (le «PTE*" y frecuencia de presencia fúugica

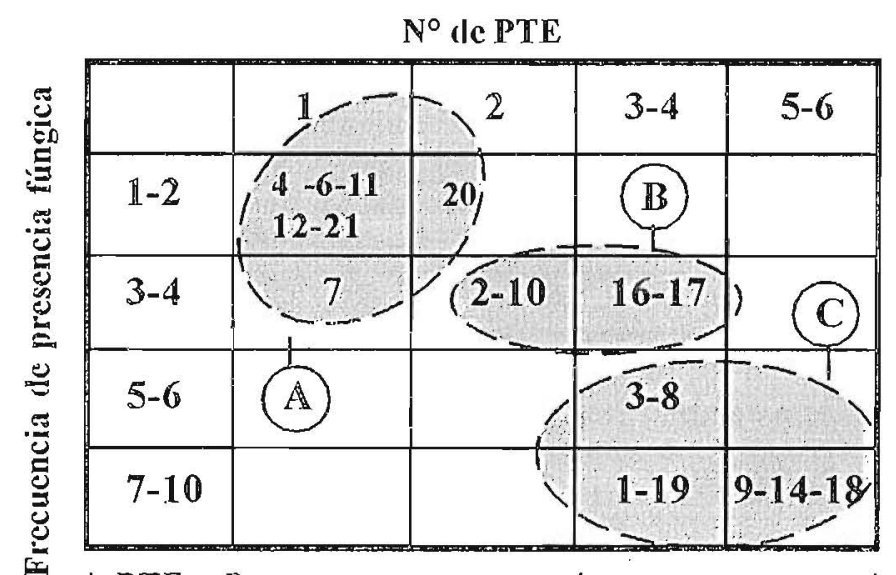

* PTE = Puntos temporo- espaciales én que se encontró la especie de graminea colonizada (represemada por. números). Cada pumlo consideró sólo la presencia en las 3 estaciones $v$ los thgares de muestreo.

(i) Datos metereológicos. Los datos melereológicos referentes a las épocas de colecta, se obluvieron del «Osservatorio Metereologico dell' Istituto Tecnico Agrário Statale C. Gallini di Voglıera» y del «Ente Regionale di Sviluppo Agricolo della Lombardia». Estos dalos se resumen en la Tabla $\rfloor$.

\section{RESULTADOS}

La menor pluviosidad y las mayores temperaturas medias se presenlaron en verano en Milano, en oloño se obtuvieron la más altas pluviosisdades en Cremona y' las más bajas temperaturas en Bergamo (Tabla J).
En 18 de las 21 gramíneas colectadas, se obtuvo la lolalidad de los aislanientos fúngicos (79), en 3 de ellas (Brachypodium pinnatum, Holcus lanatus y Lolium milliflorum) no se deleclaron representantes de los laxa considerados. Más del $98 \%$ de las especies aisladas se presentaron como anamorfos de Cochliobolis y las más representativas fueron Bipolaris anstraliensis $(30,4 \%)$, Cochliobolus sativus $(27,8)$ y C.cymodomtis $(22,8)$ (Tabla.2, Fig. 2). Voghera y Cremona fueron las zonas de mayor presencia de estas dematiáceas. Voghera presentó la mayor riqueza de especies y más del $50 \%$ del tołal do presen.cia de B. australiensis (Fig. 3, Tabia 2). Las espigas y ]as hojas fueron las más afecladas $\cdots$ ia colonización fúmgica, especialmente en verano y el culno en oloño (Fig. 2-4).

Tabla 2. Presencia fúngica por localidacl

\begin{tabular}{|c|c|c|c|c|c|c|}
\hline Especies & ${ }^{*} \operatorname{Ber}$ & $\mathrm{Cr}$ : & Mil. & Voght. & Tot. & $\%$ \\
\hline $\begin{array}{l}\text { B. australiensis } \\
\text { (Tsudia \&Ueyama)Alcon }\end{array}$ & 4 & 6 & l & 13 & 24 & 30,4 \\
\hline $\begin{array}{l}\text { Cochl. sarivus } \\
\text { (Ito \& Kurib.) Drechsler }\end{array}$ & 6 & 8 & 3 & 5 & 22 & 27,8 \\
\hline $\begin{array}{l}\text { Cochl.cynodontis } \\
\text { Nelson }\end{array}$ & 3 & 7 & 4 & 4 & 18 & 22,8 \\
\hline $\begin{array}{l}\text { B.sacchari } \\
\text { (L. Buttler) Shoem. }\end{array}$ & 6 & - & - & 1 & 7 & 8,8 \\
\hline $\begin{array}{l}\text { Cochl.spicifer } \\
\text { Nelson }\end{array}$ & - & 1 & 2 & 2 & 5 & 6,3 \\
\hline $\begin{array}{l}\text { B.homomorphliws } \\
\text { Lulli: \& Rogerson }\end{array}$ & - & 2 & - & - & 2 & 2,5 \\
\hline $\begin{array}{l}\text { Curv.protuberata } \\
\text { Nelson \& Ilodges }\end{array}$ & - & - & - & 1 & 1 & 1,3 \\
\hline Totales & 19 & 24 & 10 & 25 & 79 & \\
\hline * Berg. = Bergam & $\begin{array}{l}\text { 11o, C } \\
\text { Vogh }\end{array}$ & $\begin{array}{l}\text { Cres } \\
\text { Vogh }\end{array}$ & $\begin{array}{l}\text { mona } \\
\text { era. }\end{array}$ & Mil. & Mil & \\
\hline
\end{tabular}

Cuadro 2.- Fiecuencia (le presencia fúngica en los $\operatorname{Grupos}(\mathbb{A}, \mathbb{B}, \mathbb{C})$, considerando: estructura regetal, estación del año y lugăl (le muestreo.

\begin{tabular}{|c|c|c|c|c|c|c|c|c|c|}
\hline & \multicolumn{2}{|c|}{ Estructura } & 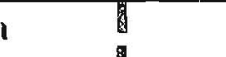 & \multicolumn{2}{|c|}{ Eslación } & \multicolumn{4}{|c|}{ Lugar le muestreo } \\
\hline & Culmo & Moja & Espiga Prim. & Verano & Otoño & Voglicia & Cremorla & Bergamed & Milano \\
\hline Grupo A & 4) & & 9 & 5 & 5 & 10 & • & & 2 \\
\hline Grupo B & 1 & 6 & 11 & 7 & I & & 9 & 2 & \\
\hline Grupo $\mathbb{C}$ & 15 & 18 & 32 & 24 & 15 & & 16 & 15 & 6 \\
\hline
\end{tabular}


Usando el criterio descrito en metodología, el grupo $\mathbf{A}$ incluye los siguientes números de gramíneas; 4- 6-7-11-1220 -21; el B ; 2-10-16-17 y el C; 1-3-8-9-14-18-19, las que aparecen sombreadas en el Cuadro 1. Cynodon dactylon, Hordeum murinum y Poa pratensis, fueron las gramíneas que presentaron un mayor grado de infestación en el tiempo y el espacio, sin embargo, en Setaria viridis se obtuvo el mayor porcentaje de aislamientos de estas dematiáceas ( $12 \%$ ) y en Hordeum murinum la mayor riqueza (5)de especies.

Al relacionar estos 3 grupos con las estructuras vegetales infestadas, con las 3 estaciones analizadas y las zonas de muestreo, se aprecia que los 3 grupos tienen la mayor presencia en la espiga en verano. El grupo $\mathrm{C}$ de mayor riesgo, se presenta con lá más alıa frecuencia (Ma- yor o igual al 30\%) en Cremona y Bergamo, no solo en verano sino también en oloño con altas frecuencias (Cuadro 2).

Las especies más frecuentes en el grupo $\mathrm{A}$, son $B$. australiensis $(41,7 \%)$ y Cochliobolus cynodontis (33,3\%), en cambio en los otros 2 grupos ( $\mathrm{B}$ y $\mathrm{C}$ ), las especie más frecuentes además de las mencionadas anteriormente fue C.sutivus (Cuadro 3).

Los datos morfométricos de los promedios del largo-ancho de los conidios y número de septos, se detalla en la Tabla 3. En general todos los taxa determinados coinciden con los rangos y los promedios de la literatura especializada, salvo en B. sacch ari donde se aprecia que el largo, supera un poco el promedio descrito.

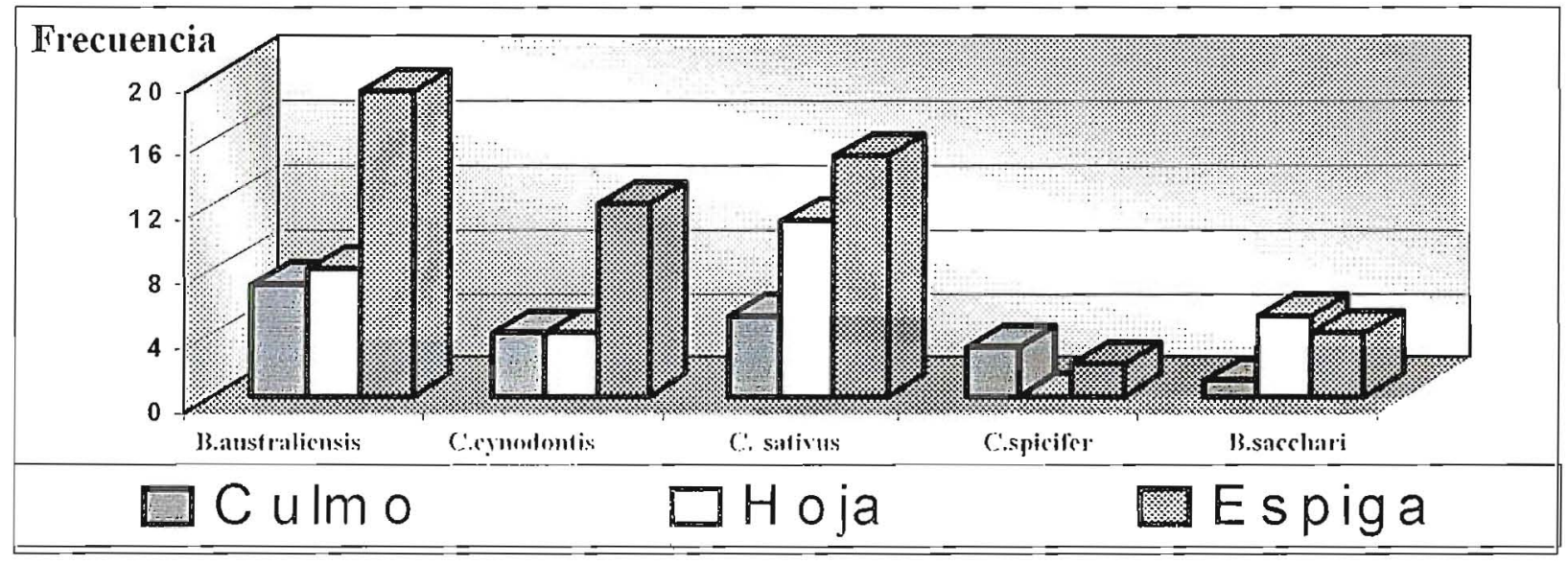

Figura 2,- frecuencia de las princiopales especies füngicas en relación a las estructuras vegetales (Culmo, hoja y espiga)

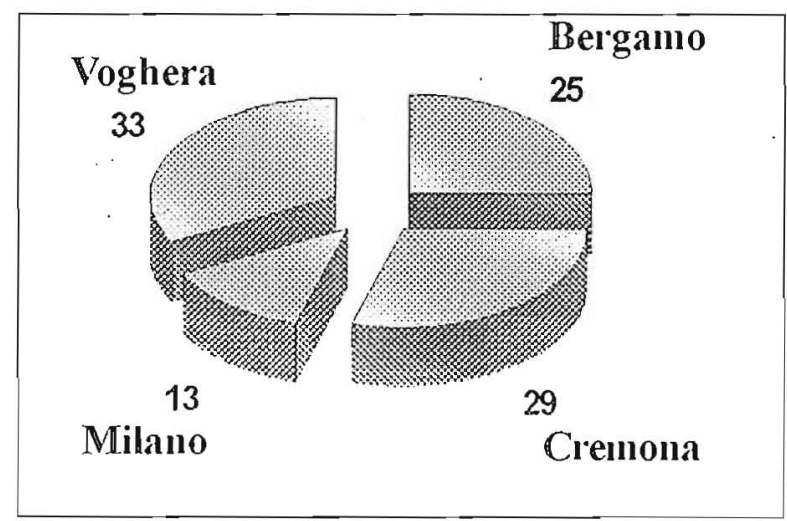

Figura 3.- Porcentajes totales de presencia según localidades

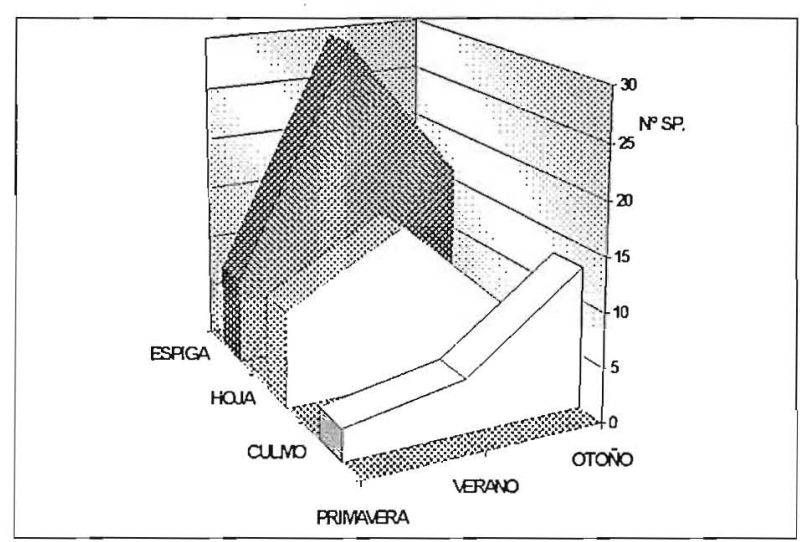

Figura 4.- Total de presencia fúngica en relación a estacionalidad y estructuras vegetales 
Cuadro 3.- Frecuencia fúngica en los grupos (A, B, C) según especies

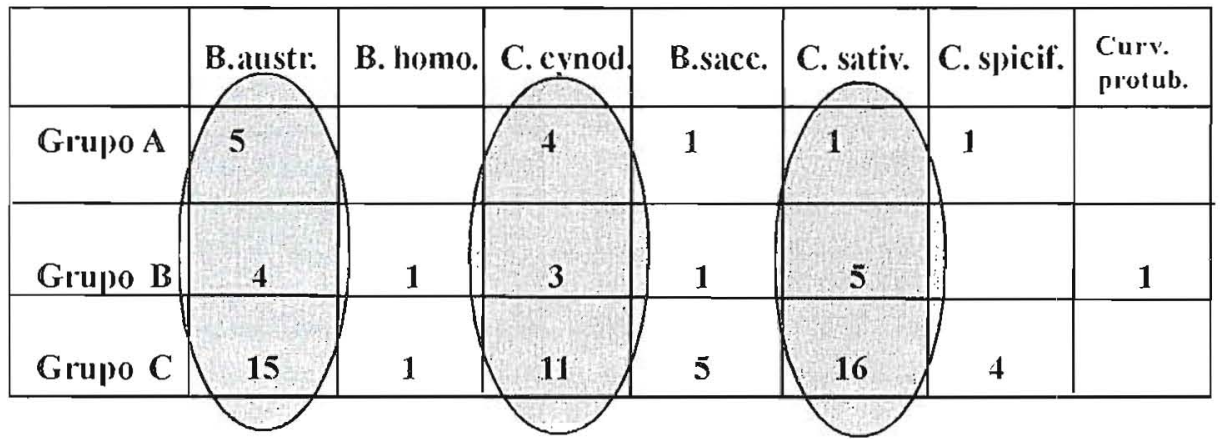

Tabla 3.- Datos moıfométricos de las especies aisladas (valores medios).

\begin{tabular}{|lll|}
\hline \multicolumn{1}{|c}{ Especies } & Conidios & $\mathrm{N}^{\circ}$ septos \\
B. uustraliensis & $29,5 \times 9,2 \mu \mathrm{m}$ & 3 \\
Cochl. sativus & $74,5 \times 26,1$ & 7 \\
Cochl. cyluodontis & $43,5 \times 13,4$ & 8 \\
B. sacchuri & $73,3 \times 14,3$ & 8 \\
Cochl. spicifer & $31,4 \times 12,5$ & 3 \\
B. homomorphus & $29,4 \times 11,0$ & $4-5$ \\
Curv. protuberatu & $30,5 \times 11,8$ & 3 \\
\hline
\end{tabular}

\section{DISCUSION}

De los géneros graminícolas integrantes del complex Helminthosporium sensu laro. sólo delectamos en estas gramíneas silvestres. la presencia casi absoluta de representantes mitospóricos del género Bipolaris, un taxon conocido por sus capacidades necrol róficas y causante de diversals sintomatologias en raices, loọas, culmos. inflorescencias y semillas de varios cereales. Bipolaris sorokiniana, B. ma!diis, B.oryzac, B.cymodontis, B.spicifera, B.halepense, entre otros integrantes de este gran género, se presentan ya sea en trigo. sorgo, maíz, avena u otras gramíneas forrajeras como hospedaclores alternativos, mantenienclo variados niveles poblacionales en el suclo (Wildermu1, 1986: Wildermut \& McNamara. 1987; Sisterna, 1087: Sonocla, 1991: Cappelli et al. 1993: Liljerolh et al., 1996: Carson. 1998).

Comprender la variación temporo-espacial en las poblaciones fúngicas: tiene gran relevancia porque permite conocer la biodiversidad y los roles que los hongos tienen en la regulación de las poblaciones de otros organismos (epífitos. endógenos y palógenos) y en los diferentes procesos del ecosistema. Los hongos patógenos que afeclan las superficies vegetales varían en respuesta al incremento de la clensidad de sus hospedadores. con ribuyendo a la clispersión y la riqucza de especies en la zona. en la descomposición de los nutrientes cíclicos y al mismo tiempo al aumento de la heterogeneidad espacial de las comunidades descomponedoras (Lodge \& Cantrell, 1995).

Lá diversidad de gramíneas estudiadas. incluyó tanto a taxa presentes en jardines, bordes de áreas cultivables, caminos, colonizadoras o infestantes de praderas o cultivos, como a especies de uso forrajero (Pignatti, 1982). A pesar de su variedad, la vegelación leerbácea de las áreas analizadas, engloban aspectos ecológicos, nutricionales y biogeográficos bastante similares. ligados principalmente al tipo de suelo, humedad ambiental, disturbio provocado por la alta densiclad poblacional y a las aciividades agrícolias caracteristicás de lás zonas adyacentes. Por sus capacidacles adaptalivas y competitivas, pueden colonizar ambientes fuertemente degradados (Duke, 1985). e incluso el rol polencial de algunas especies de gramíneas en el biodetcrioro, debe considerarse en la consevación de bienes monumentales y arqueológicos (Signorini, 1996).

Como grupo taxonómico, las gramíneas silvestres y cultivadas, más que las leguminosas, son hospedacloras de una gran variedad de hongos dematiáceos que actúan. ya sea como colonizadores o infeslantes, en semillas, raices, follaje e inflorescencias, especialmente en sus períodos de senescencia. Esta última siluación se aprecia en la capacidad de colonización de los 3 grupos cle especies fúngicas $(A, B$ y $C$ ) , principalmente en verano y oloño, sin cmbargo. el grupo B. parece más propenso a colonizar tejidos jureniles. Las estructuras senescentes generalmentc contienen baja cantidad de azúcar, debido a la pérdida de clorofila, una reducida fotosíntesis y un aumento de la respiración. La disminución de la cantidad de azúcar en las hojas. ha permitido sugerir un anmento de manchas foliares por C. sativus en Poa pratensis (Madsen \& Hodges. 1983).

Segúm el criterio metodológico empleado son 7 las principales gramineas (Agropyron repens, Arrhenather'm!n elatium, Bromus sterilis, Cynodon dactylon, Hordeum murirum, Poa pratensis y Setaria viridis) que presentaron un mayor grado de colonización en el liempo y cl espacio. Bajo el punto de vista fitopatógeno, estas gramíneas no sólo representan un reservorio fúngico latente y resis- 
tente al estress (Muchovec, 1986; Duczek, 1994), sino un inóculo no despreciable de fitopatógenos importantes para la dispersión en áreas cercanas de la Lonbardía, donde abundon otras gramineas cultivadas susceplibles de infestarse (trigo, maz, arroz, cebada, etc.). Las rohoiones hongo-vegclal, a veces cspecificas en los integrantes del géncro Bipolaris, podrian además tener un particular interés en el control biológico (mico-herbicida) cn la lucha contha estas gramineas silvestres infestames, en zomas donde no existan otros hospedadores susceptibles de interés agronómico (Wilson, 1969; Winder \& Dyke, 1990; Vurro \& Bollalico, 1993).

Un buen numero de especies fungicas en estos taxa, son capaces de producir fitoloxinas con roles primarios en el desarollo de una particular patología o la muerte de los tejidos vegetales en muchas gramineas infestantes como B.cynodontis (Bipolarotoxina) B.sorghicola, B3. mardis (Ophobolina), B.sorokiniant (Prehclminthosporol), Ahernaria tenuis (Tentoxina) y Alternaria alternata aggr.(Maculosina, alicmariol, ctc). (Vurro of at. 1992: Lilierol et al., 1996). Esta úllima especic a pesir que no pertenece al grupo fingico seleccionado on muestros objelivos, fic la especic más dominante en liempo y espacio en todas las gramincas estudiadas (datos no publicados). Is.rustrationsis, C.stativers y C.cyodontis, mucstran una distribución bastanle similar en lodos los lugares de muestreo y $\mathrm{cn}$ los grupos, lo que puede atribuirse a sus amplios rangos de hospedindores regelales la mayor competitividad. que junto al menor disturbio (las árcas no cultivadas donde crecen estos pastos), permiten una mayor sobrevivencia y adaplación de sus poblaciones en los residuos vegelales del snelo y en tas semillas de estas gramíneas (Celetti, 1990; Capclli,1991, 1993).

Como comenta Montorsi et al.(1988), las condiciones climálicas del norte de Inlia, parecen ser más favora- bles al desarrollo de varios palógenos fúngicos de gramínens que las zonas centrales y septentrionales del pais. La presencia de especies de Bzipolaris u olros representan\{es del "complex Ifelminthosporinun en variıș gramineas cullivadas, forrajeras o cn sus semillas, se ha detoctado cn diversas regiones de lialia (Govi et al., 1974; Montorsi ef al, 1988; Cappelli, 1991). En cl norte de la península, sc destuca la presencia de B.oryzae cn arroz, cn las zonas cercanas a Voghera (Molettiet al, 1996), Fyrenophorateres en cebada, cn la zona de Piacenza (Cornzza et al., 1988) y cn la zon central (Unbria), Csativas en trigo (Rossi et al., 1091: Cappelli et al, 1993; Capelli, 1993). C. sativus, causa manchas foliares, marchilamiento de raíces y tallos, ocasionado muchas perdidas en pastos, trigo y otros granos pequeños en muchas árens del mundo (Salvinder et al., 1920: Wildermuth, 1286; Sistema, 1987; Piontelli \& Grixolli, 1994). La fase foliar de la cnfermedad puede ser conómicamente importante sólo en áreas con alta humedad y calurosas (Bilcs \& Hill, 1988; Liljerol et al, 1996), wha siluación bastante común en Lombardía en ciertas estaciones del año, permiticndo un aumento de sus poblaciones en el suclo y la vegetación circundante, así como de B.anstralicnsis y C.cynodomis.

La densidad poblacional de estas 3 últimas especies además de C. spicifer en las 4 áreas muestreadas, debe considerarse no sólo desde el posible aspecto potencial fitopatológico regional en varias gramincas silvestres o cultivadas, sino también, desde un punto de visla de patologia humana, donde estas dematiáceas han causado problemas alérgicos o sistémicos en la población (Piontelli \& Grivolit, 1994: Palacioet al., 1997; Noble et at. 1997).

Agradecienientos.

Se agradecen las sugerencias y útiles comentarios aportados por el Prof. Dumny Casanova.

\section{REFERENCIAS}

Aleon, J.L. (1982). Oraricolous Bipolaris species on Sporobolus and other grasses. Mycolation 15:20m48

Alcorn, I.L. (1983). Generie Concepts in Drechslera, Bipolaris and Exserohilmm. Mycoluxon 17:1-86

Alcorn, J.L. (1920). Additions to Bipolaris, Cochliobohs and Curmlaria. Myoulaton 39:361-392

Alcorm, M.L. (1991). Now combinations and synonym in Bipolaris and Corvitarin, and a new species of Exserohilum. Nycotaxon $41: 329-343$

Biles, C.L. \& Ilill, J.P. (1988), Elect of Trichodema harianmm on sporulation ol Cochliobolns sativus on axeised wheal seedling lates. Plyytopathology 78:656-659

Cuppelli, C. (1901). Presenza di Drechslera siccans (Dreih.)
Shoemaker e D. andersenii Lam in sementi di loietto (Lolinm perenne L.). Sementi Elate 37: 49-51

Capelli, C. (1993). Indagini sulla volpaturn delle eariossidi di Trmento duro prodolte in alome locilita dell'Abruzzo nel 1990. 91. Sementi Elette $40: 29-35$

Capelli, C.; Buomario, R. \& Ciriciofolo, E. (1993). La puntutura del frumento duro in Umbria. Un Biemio di osservazioni. Inlormatore Fiopalologico 11:24-28

Carson, M.L. (1998). Agressiveness and permmation of isolates of Cochtiobohs heterostrophus from Norh Carolina. Plant Dis. $82: 1043-1047$

Celeti,M.J. (1990). lncidence of soil-bome plant pathogens isoluted from barley and winter whent, and other crops in the rotation, on Prince Edwards Jsland. Plan Pahology 39:600-611 


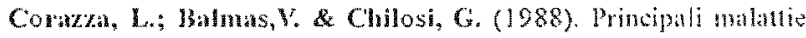
fungine dell orzo osservate in sampo nel 1287.88 . Inormatore Agrario $33: 67-71$

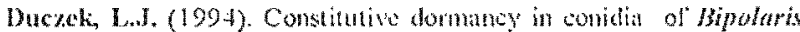
sorokinama. Can. I. Plan Pallol. 16:347-349

Duke, 5.0 . (1985). Reproduction and Ecophysiology Nexd Physiology $1: 133$

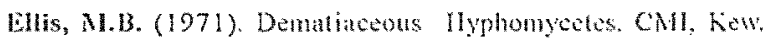

Ellis, M.B. (127). More Demahineous Hyphonycetes. CMI, Kew

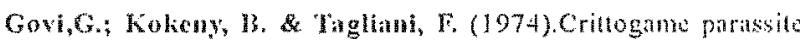
di graminace foraggers. Inlomatore Filopalologico 24 (3): $21-26$

Korf, R. (1991). Shall we abatudon the principle of priority (and oher nomenclatuml caveats) for the sale of expedicney ?. Mycolation 40:459-468

Lim, A. (1982). Presence of Drechslera specios in certhed ryegrass sexd lots. Grass and Forage Scisnee 37:47.52

Lam, A. (1983). A surey of yegrats swirds lor low presnes of fungal pathogens in relalion to managenent. Grass and Fomgo Science $38: 55-65$

Liljeroth, E.; fommon-thmgrem, I Gunnussun.T. (1996).

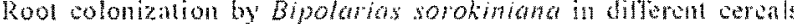
wnd pelations to bsion depelopnent and matul root cortical call death. 1. Phylonathology 144; $301-307$

Lotge, D.J, R Cantrell, $\$$. (1925). Tungul communities in wet lopical forest: vatition in time and spate.Can. I. Bol.73 (Stipl.1): $\$ 1391-1398$

Matisen, J.1. \& Houges,C.I. (1983). Ellect of chlorophenoxy herbicides on soluble sugars and on pathogenesis by brechsturn sorohintma in sequentidly sentecen leaves of pot phatensis. Phylopathology $73: 1296-1292$

Nolett, M. (1989). Acune malatis del riso in Italia. Intomnton Fitopatologico $39: 29-36$

Moloti, M.; Gintle M.L. \& Villa, D, (1988-92), Valutazione della ronzione di alcuni genotipi di riso all blmintosporiosis. Centro di Ricerche sul Riso. Ente Naziona d kisi. Relazione sullattiviti sqolla nel 1988:46-47; 1989:51-53; 1990:58-61; 1991:8-10; $1992: 8-10$

Moleti, M.; Gudici,M.L, \& Vha, B. (1996). Lukiochiemmosporiosis det riso in llalin: Lota agronomica s chimica Inlormatore litopatologico $6: 41-46$

Montorsi, Fo: Pezalt, M.d Margarita, L. (1988), Alcune ossentaioni sullo stuo smitaro di sememe di grono duro in latha Settentriomale. Intomatore Fitopatologico 10,63-67

Muchover.s.s. (1986). Delinition of two healh in agrosis palustris, at the time of indections and colonisation by Curvatarit hmora. Ammis of Applict biology 109:249-258

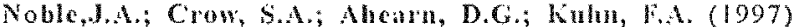

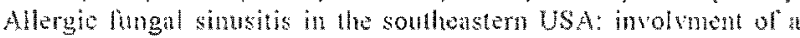
new agent Epicaccum nigram Ehremb. Ex Sehlecht. 1824. I. Wod Vet. Myeol. $35: 405-409$

Nuter, F.W. (1982). Condial sampling of Drechstera pode from Kentucky bluegrass to delemine role ol mowing in spore dispersal. Plan Dis $66 \cdot 721-723$

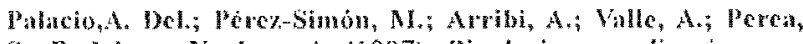

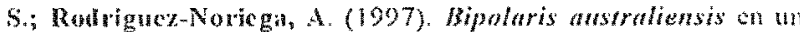

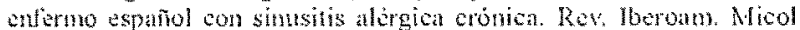
$14: 191-193$

Pjganti, \$. (1982). Flota t'llatia. Eangricole, Bologna.

Piontelti, L. Grixoll, M.A. (1924). Especies gramincolas de Bipolaris Drechstara y Curvilaria en ha $\mathrm{V}$ Region (Chile): Aspectos morlologkeos yiogeograticos. Boletin Micologico 9:87.97

l'orta-l'uglin, A. \& Del Serrone, P (1991).A ncw spectes of Drecistert on lohusongrass. Titopal. Madit. $30: 164=166$

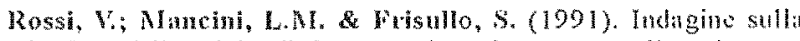

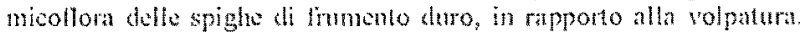
Amm. Fac. Agratia Piacenza 30:125-133

Satvinder,K.; Mann, A.P.S. \& Aulakb, K.\$.(1900), Pahogenio potential of seven Drechstera species prevalent in ceral seods. Indian Plytoputh. $43: 179-185$

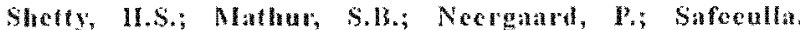
K.M.(1982) Drechster sethid in Indin pearl millet sed, it sextbone mure, transmission and significance. Truns. Br.myeol.5oc. $78: 170 \times 173$

Signorim, Mat. (1996). I, indice di Pericolositain contributo del botmico al controllo dellh regetazione inlestante nelle aree monumentali. Int'. Bot. Mal. 1: 7.jt

Sisterna,M.(1907). Especies grminicolas de los generos Dre

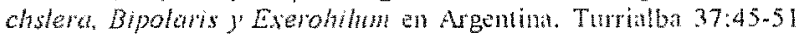

Sivanesun, A. (1987). Grammivolous species of Brpolaris.

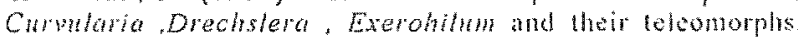
Meological papers $N^{\circ}$ is\&, CAB Intermational Hycologionl Instituto - Kew.

Somota, R.M. (1991). Exerohnhm rowtihm and Bipolaris hanetents causing lent and eulm lesions on Callide Rhodesgrass. froceding- Soil and Crop Science Sociely of Florida $50: 28-30$

Thomas, M.R \& Shattock, R.C. (1986). Filamentons fungal associntions in the plytloplane of Loliwm perenne. Thans. Br. mycol Soc. $87: 255-268$

Vurro, M. Zomo, M.C. \& Bollalico, A. (1992). Sul polenziale inpiego di Sephorin cirsii come nieorbicida conto Cismm anente. In:Alli "Giomate Filopatologiche $1922 \% 3,189-198$

Vurro, M. Botalico, A. (1923). Funghi fitopatogent per h lota alle malerbe. Infomatore Fitopnologico $4: 21-24$

Whinder, R.S. \& Dyke, C.G. Yh. (1900). Fielt lesing of two Bipolaris species as mycoherbicide for johmongrass (Sorghum halmpense) In ibrocedings of the Vlll iner. Symp. on Biologica Contr of veds Roma pp.565.570

Willermuth, G.1B,(1986). Gsographic dismbution of common root rot and Bipolatis sonokiniand in Questand when solk. Austratian I. Exper, Agt 26:601-606

Withemuth, G.B. \& MeNomatr, R. 13. (1987), Susceptibility of winter and summer crops to rool and cown infection by Bipolnts sorokinama. Pant l'athol, 36:481*491

Wilson, C. L. (1969). Ust of plan pathogens in tyeed control Am. Reve Phylopat. $7: 411-434$ 

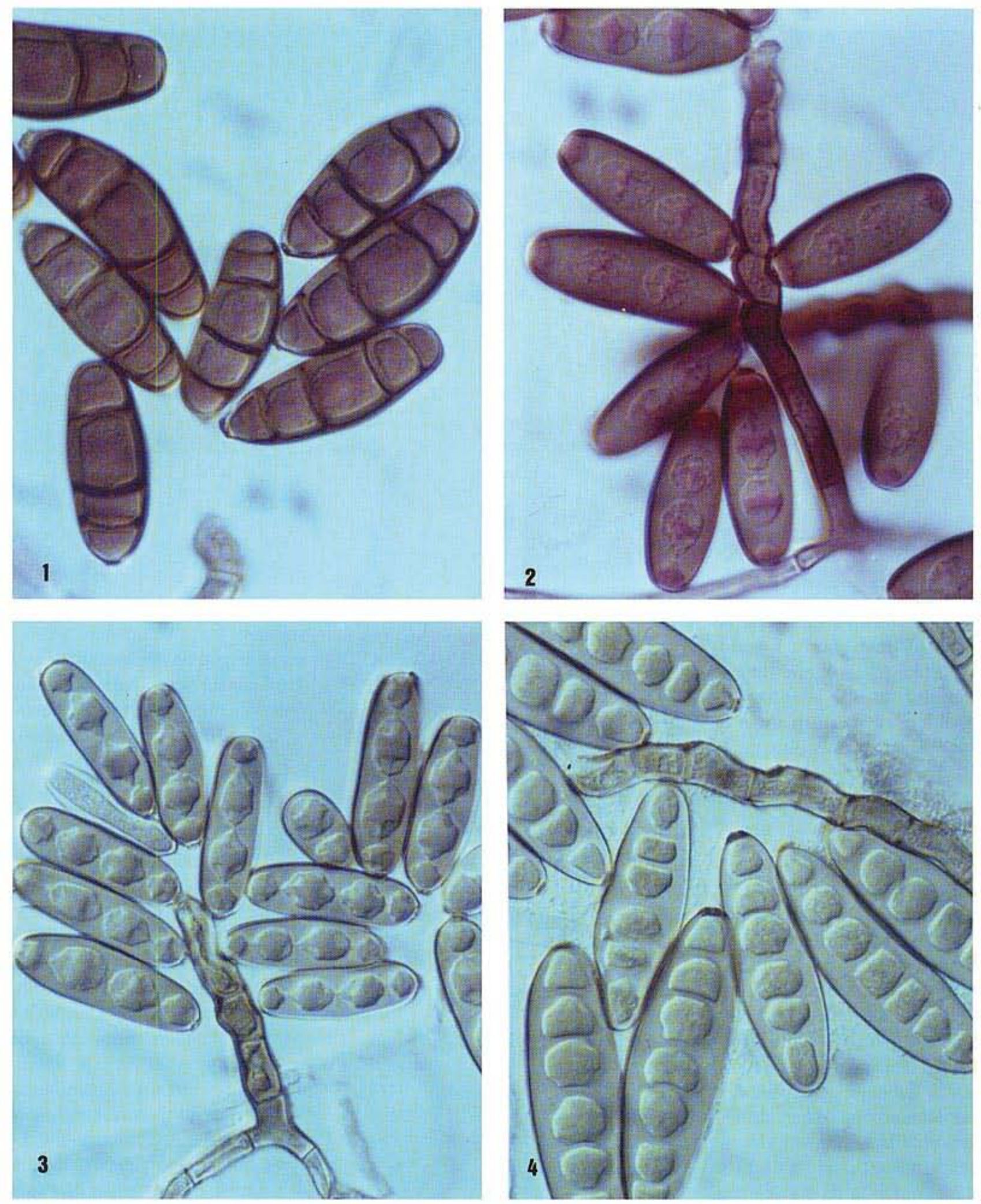

Fotografías 1-4.- 1.- Curvularia protuberata, conidios mostrando la presencia de hilum 1000X. 2.- Cochliobolus spicifer, conidios y conidióforos $1000 \mathrm{X}$. 3.- Bipolaris australiensis, conidióforos y conidios 1000X. 4.- Cochliobolus cynodontis, conidióforos y conidios $1000 \mathrm{X}$. 

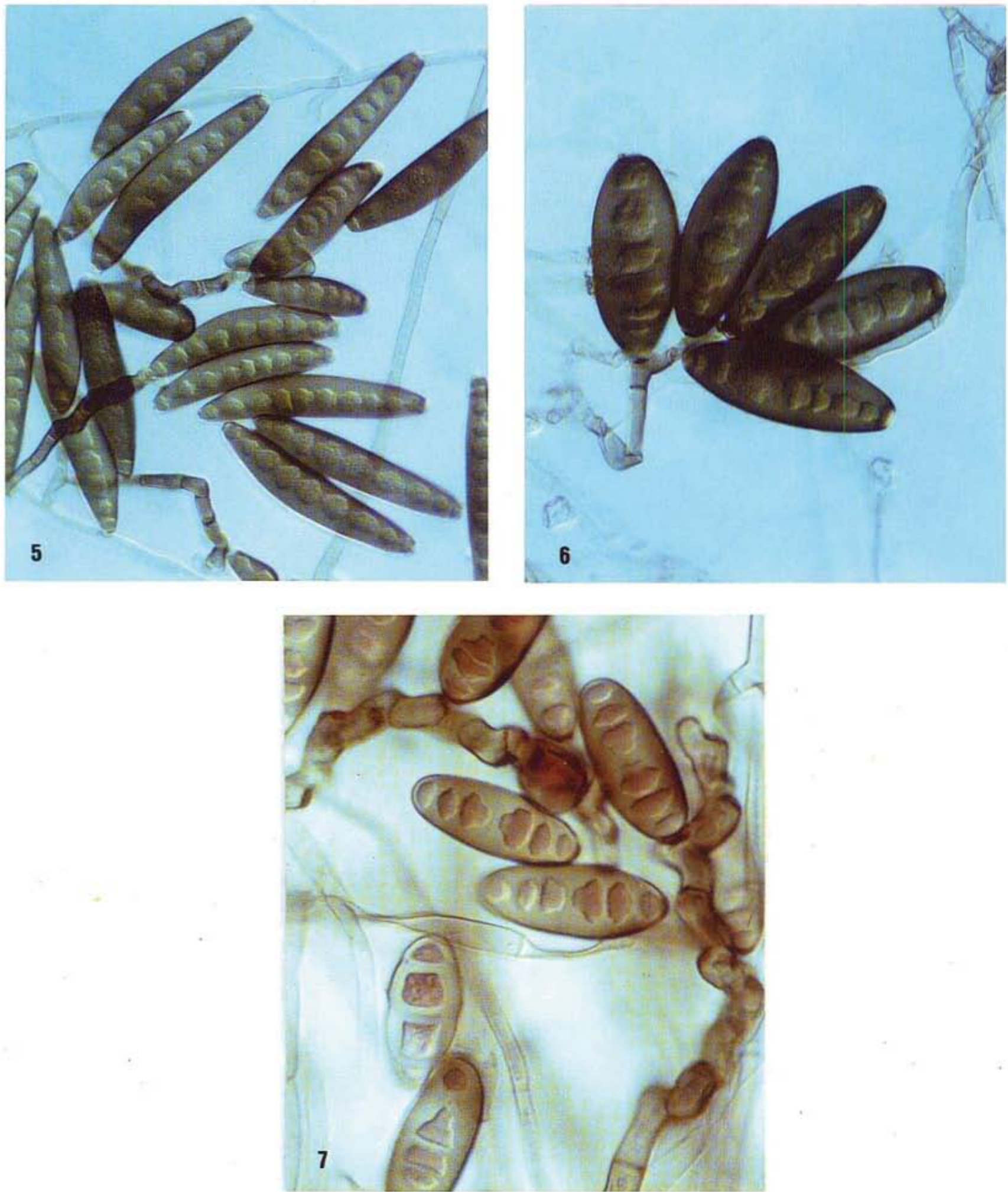

Fotografias 5-7.- 5.- Bipolaris sacchari, conidios y conidióforos $400 \mathrm{X}$. 6.- Cochliobolus sativus, conidios y conidióforos 400X. 7.-Bipolaris homomorphus, conidióforos y conidios $1000 \mathrm{X}$. 